\title{
ETUDE THEORIQUE DE LA REPARTITION DE LUMINANCE SUR LE DISQUE DE VENUS
}

\author{
C. BIGOURD, J. L. DEUZE, C. DEVAUX, M. HERMAN, et J. LENOBLE \\ Laboratoire d'Optique Atmosphérique, Université des Sciences et \\ Techniques de Lille, France
}

\begin{abstract}
The analysis of the sunlight scattered by Venus gives some insight upon its clouds. The measurements of polarized light are probably more sensitive to the nature of their constituents, and some recent studies seem to be able to give a satisfactory interpretation of this part of the scattered light. But the polarized light concerns the upper part of the clouds, and it is interesting to compare these results with intensity measurements. The phase curves, for the integrated light, leave some indeterminations, so we have studied if the intensity distribution on the Venus disk could give more accurate informations. This work, based on some plates kindly communicated by A. Dollfus, and analysed at Meudon Observatory, is more a preliminary investigation of the sensitivity of the method than an interpretation of the partial results presented. A simple model, of homogeneous plane parallel cloud, has been used, and the influence of various parameters has been tested (single scattering albedo, refractive index of particles and size distribution, optical depth of the cloud).
\end{abstract}

Nous avons étudié la distribution relative, sur Vénus, de la lumière solaire diffusée. Ce travail a été entrepris en collaboration avec le Professeur Dollfus, qui nous a communiqué quelques réseaux expérimentaux des courbes d'égale luminance, ou isophotes, déduites des clichés de Vénus. On ne poussera toutefois pas la comparaison entre ces réseaux et les calculs, les clichés devant encore être déconvolués des effets de diffraction et de la turbulence atmosphérique, avant leur publication définitive. On considérera donc plutôt ce qui suit comme une étude préalable de ce que peut apporter ce type d'analyse. L'idée est que la luminance, beaucoup moins sensible que la polarisation aux propriétés du milieu, permet par contre un sondage nettement plus pénétrant du nuage.

Nous supposerons d'abord l'épaisseur optique infinie, et partirons du modèle déduit par Arking et Hansen (1971), des mesures de polarisation; soit un nuage de particules sphériques, d'indice 1,46 environ, et de granulométrie de la forme

$$
n(r)=n_{0} r^{p_{1}} \exp \left(p_{1} r / p_{2}\right), \quad p_{1}=6, p_{2}=r_{c}=0,8 \text { à } 1 \mu
$$

dont on peut déduire la fonction de phase, sous la forme

$$
p(\theta)=\sum \beta_{l} P_{l}(\cos \theta) .
$$

Il reste à préciser l'albédo pour une diffusion, des particles, $\omega_{0}^{\infty}$. Les mesures d'Irvine (1968) donnent un albédo sphérique $A^{*}$ de 0,9 environ, pour la longueur d'onde 0,58 $\mu$ correspondant aux clichés étudiés. On en déduit facilement $\omega_{0}^{\infty}$ à l'aide de la formule approchée de Sobolev, ou de celle établie par Wang (1972), dans l'approximation du noyau exponentiel, soit

$$
\omega_{0}^{\propto}=1-\frac{\left(3-\beta_{1}\right)\left(1-A^{*}\right)^{2}}{4\left(1+A^{*}\right)^{2}}=0,9994 .
$$


On peut alors évaluer la luminance diffuse (on suppose la géométrie plane applicable). Le calcul est fait suivant la méthode des Harmoniques Sphériques en quelques centaines de points sur le disque (de 200 à 500 points suivant l'angle de phase considéré). L'exploitation reste raisonnable, l'essentiel des calculs ne dépendant pas du point considéré, ou ne dépendant que de la direction d'incidence. On a ainsi pu étudier l'influence des paramètres du milieu sur les réseaux d'isophotes.

La figure 1 montre à titre d'exemple les résultats, pour une absorption variable,

$A L P H A=75^{\circ}$

ISOPHOTES

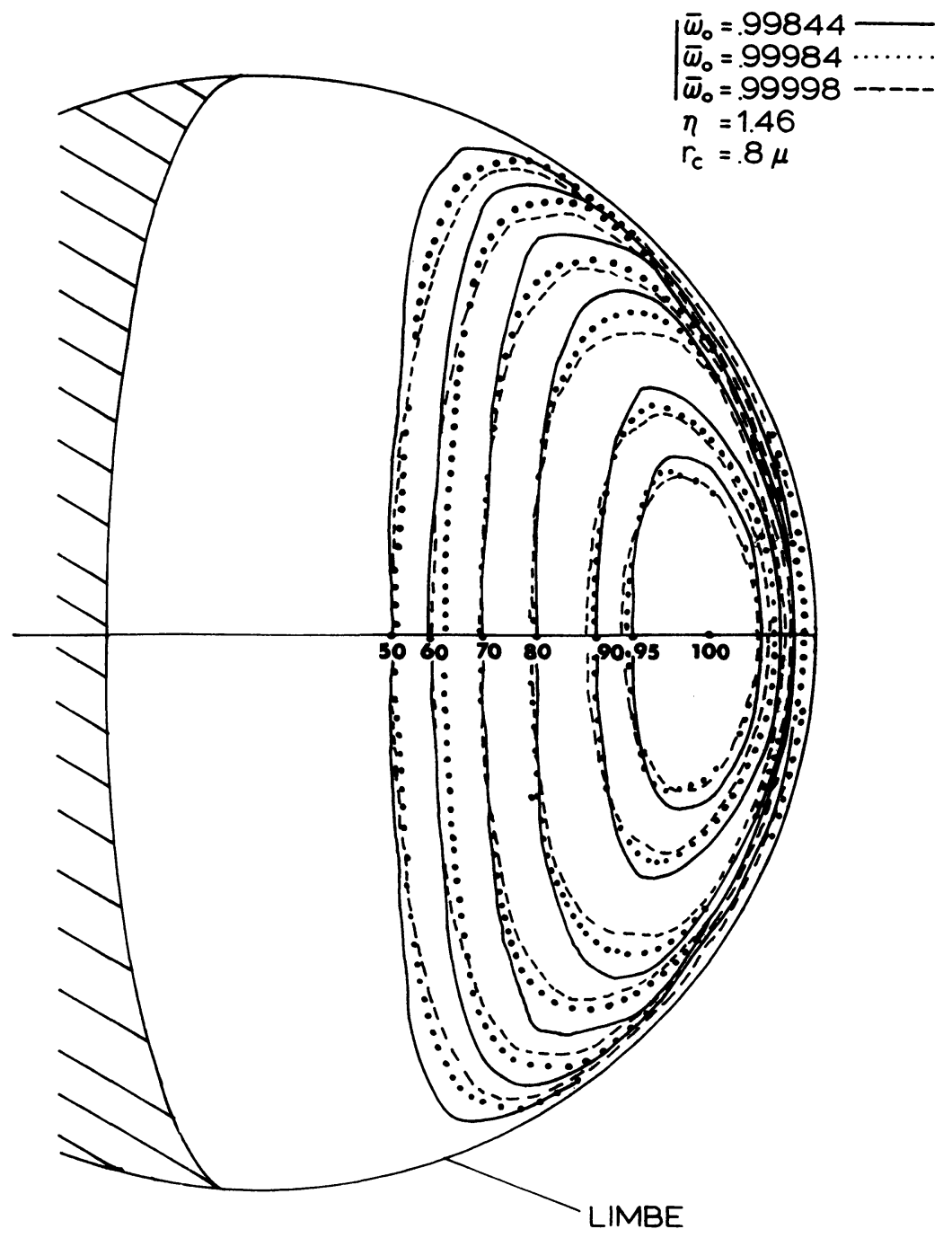

Fig. 1. Influence des paramètres du milieu sur les réseaux d'isophotes pour l'angle de phase de $75^{\circ}$. 
dans le cas d'un angle de phase $\left(\alpha=75^{\circ}\right)$; les valeurs extrêmes de $\omega_{0}^{\infty}$ donneraient des albédos sphériques de 0,64 et 0,94 , qui encadrent nettement la valeur probable (les maxima des réseaux sont toujours ramenés arbitrairement à 100). Les luminances relatives varient très peu. Pour une variation de $r_{c}$ de 0,8 à $0,3 \mu$ les modifications sont du même ordre, bien qu'on se déplace vers les petites particules, ce qui déforme au maximum la fonction de diffusion. Enfin, on a comparé au modèle d'indice 1,46 un milieu d'indice 1,20. Les écarts s'accentuent quand $\alpha$ décroît (avec l'influence croissante de la rétrodiffusion, par laquelle se différencient surtout les deux milieux); mais ils restent encore faibles, et finalement, aucun des 3 paramètres ne modifie radicalement le phénomène. On met donc bien en évidence la très faible influence des propriétés optiques du nuage sur la luminance relative.

Tenons compte maintenant de l'épaisseur $\tau_{1}$ du nuage, et donc, simultanément, de la nature du sol; on supposera simplement qu'il suit la loi de Lambert, avec un coefficient de réflexion $\varrho$. Pour fixer les idées, nous avons utilisé les résultats de Venera 8 , et tenté de déterminer, suivant la valeur supposée de $\varrho$, les ordres de grandeur de $\tau_{1}$ et $\omega_{0}$ compatibles avec les valeurs mesurées de $A^{*}$ et du flux solaire transmis au sol, au point d'impact de Venera 8 (Marov et al., 1973). Si on se donne $\varrho$ et l'indicatrice des particules, la solution du problème est unique; en remontant le fond, on ne maintiendra en effet un flux réfléchi constant qu'en diminuant l'absorption propre des particules, qu'on devra simultanément augmenter, au contraire, pour conserver un même flux transmis; d'où 2 courbes $\tau_{1}\left(\omega_{0}\right)$ qui se couperont si les mesures et l'hypothèse sur $\varrho$ sont cohérentes. On peut obtenir une solution analytique approchée du problème. On a pour cela développé, dans l'approximation du noyau exponentiel, les calculs de l'albédo sphérique $A^{*}$, du flux plan transmis au sol $\phi^{-}\left(\tau_{1}\right)$ (et du flux remontant $\phi^{+}(0)$ ), pour la géométrie considérée (Bigourd et al., 1973). La validité des formules obtenues a été testée. Si on suppose $\omega_{0} \sim 1, \tau_{1} \gg 1$ et $(1-\varrho)>0,5$ environ, ce qui doit être raisonnable, on peut déduire de ces expressions de $A^{*}$ et $R=\phi^{-}\left(\tau_{1}\right) / \mu_{0} f(f$ est le flux solaire)

$$
\begin{aligned}
& \operatorname{th}\left(C \tau_{1}\right)=\frac{1-\left[(1-\varrho) \sqrt{\left.\left(3-\beta_{1}\right)\right] /\left[(1+\varrho) \sqrt{4\left(1-\omega_{0}^{\infty}\right)}\right]}\right.}{\sqrt{\frac{\left(1-\omega_{0}\right)}{\left(1-\omega_{0}^{\infty}\right)}-\left[(1-\varrho) \sqrt{\left(3-\beta_{1}\right)}\right] /\left[(1+\varrho) \sqrt{4\left(1-\omega_{0}\right)}\right]}} \\
& \operatorname{sh}\left(C \tau_{1}\right)=\left[\left(3 \mu_{0}+2\right) \sqrt{\left(1-\omega_{0}\right)}\right] /\left[R(1-\varrho) \sqrt{\left.\left(3-\beta_{1}\right)\right]},\right.
\end{aligned}
$$

où

$$
C=\sqrt{\left(3-\beta_{1}\right)\left(1-\omega_{0}\right)} \ll 1
$$

et l'on déduit facilement de ces équations que, pour la solution cherchée, on a, en fonction de $R$ et de $A^{*}$, avec la relation (1)

$$
\omega_{0}=\omega_{0}^{\infty}+\frac{\left(3-\beta_{1}\right)(1-\varrho)^{2} R^{2}}{\left(3 \mu_{0}+2\right)^{2}}
$$

et donc $\tau_{1} \operatorname{par}(2)$ ou (3). Nous nous sommes contentés d'un calcul à la seule longueur 
d'onde 0,63 $\mu$, correspondant au maximum de sensibilité de la cellule de Venera 8. Avec $\mu_{0} \sim 0,1, R \sim 1 \%$ et $A^{*} \sim 0,94$ (soit $\omega_{0}^{\infty} \sim 0,9998$ ) on obtient

$$
\begin{aligned}
\varrho & =0 & & 0,5 \\
\tau_{1} & =50 & & 200 \\
\omega_{0} & =0,999816 & & 0,999804
\end{aligned}
$$

Ces valeurs sont à considérer avec réserve, compte tenu des approximations et des incertitudes de mesure, mais avec de tels ordres de grandeur, il serait inutile de calculer

ALPHA $=75^{\circ}$

ISOPHOTES

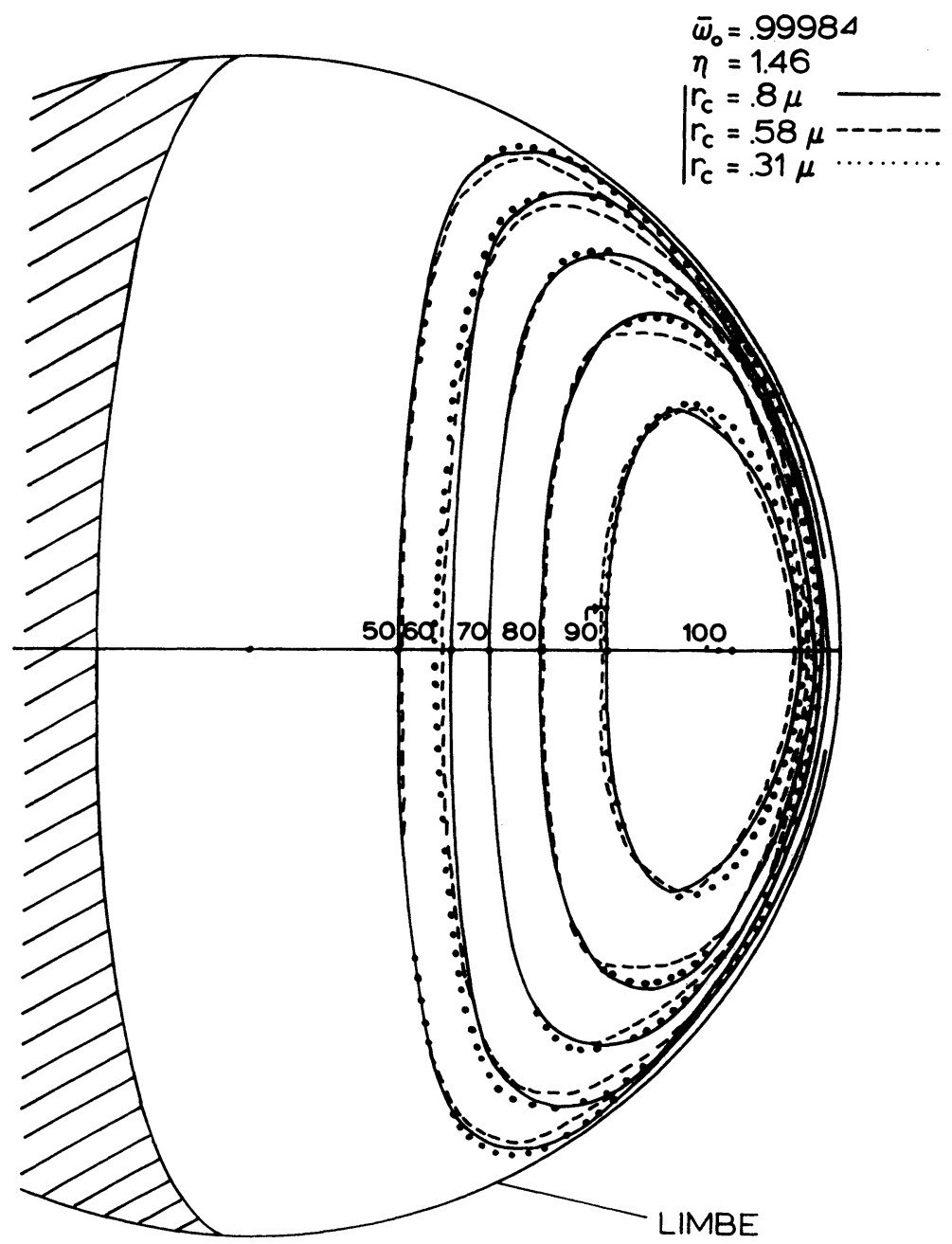

Fig. 2. Comparaison des réseaux d'isophotes obtenus pour divers diamètres. 
les nouveaux réseaux d'isophotes. Dans l'approximation précédente, en effet, le flux réfléchi en un point du disque s'écrit

$$
\frac{\phi_{(0)}^{+}}{\mu_{0} f}=1-\frac{3 C\left(3 \mu_{0}+2\right)}{2\left(3-\beta_{1}\right) \operatorname{th}\left(C \tau_{1}\right)} .
$$

La variation relative de ce flux, lorsqu'on passe d'une couche infinie à une épaisseur

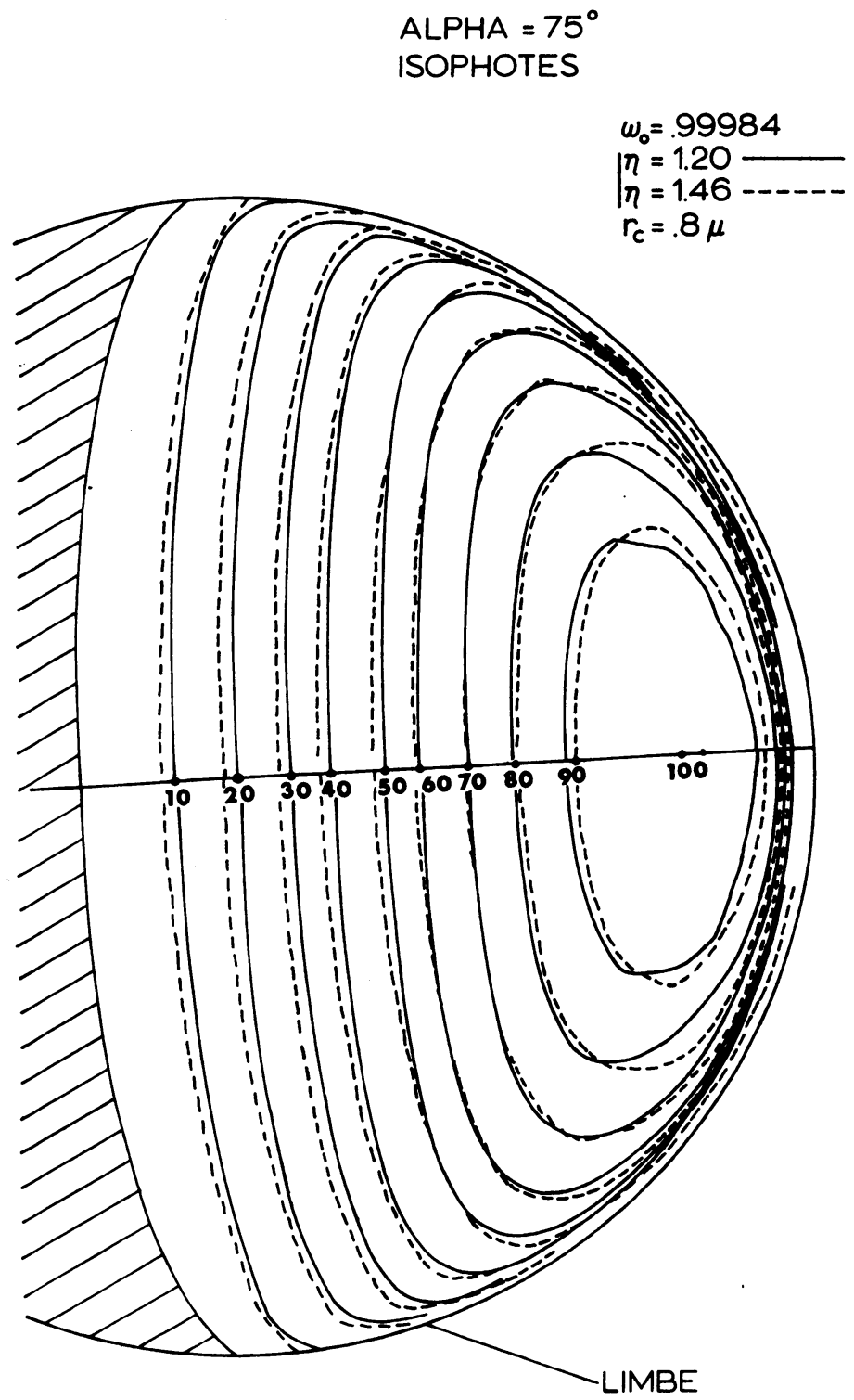

Fig. 3. Comparaison entre les réseaux d'isophotes théoriques pour $n=1,20$ et 1,46 . 
de l'ordre de 100 , ne dépasse alors pas $0,5 \%$ au point subsolaire où elle est maximum. La luminance varierait dans le même ordre de grandeur, et les réseaux seraient indiscernables.

En conclusion, si nous supposons que les nuages conservent sur toute la planète, les caractéristiques déduites de la polarisation, et une épaisseur supérieure ou égale à celle qu'on peut envisager au point sondé par Venera 8 , la distribution de luminance relative est calculable avec précision, et une comparaison précise devrait être possible.

Si l'on s'interroge sur la possibilité de détecter la présence éventuelle, sous la brume sondée en polarisation, d'un nuage de nature différente mais d'absorption propre voisine, la conclusion semble négative. La figure 3 montre l'ordre de grandeur des écarts maxima observés pour un nuage d'indice 1,20 au lieu de 1,46 , dans le cas où $\alpha=22^{\circ}$. Ces écarts seraient encore atténués par la superposition d'une couche, même fine, d'indice 1,46 . De plus on doit être à la limite de la précision des mesures. (Il s'agirait de mesures diurnes, et les faibles niveaux de luminance, ou se localisent justement les plus gros écarts, seraient perturbés par le voisinage du soleil).

Il n'est pourtant pas exclu que ces mesures présentent une intéressante extension. Les comparaisons entre réseau théorique et expérimental montrent des. divergences qui paraissent très fortes, et on peut se demander si la correction des effets de turbulence et de diffraction pourra suffire à les effacer. D'autres clichés présentent par ailleurs de nettes dissymétries équatoriales, et parfois même des isophotes fermées sur un seul hémisphère. Il peut donc se révéler nécessaire d'élargir le modèle en introduisant une inhomogénéité horizontale. Pour qu'elle soit capable d'influencer la répartition de luminance relative, compte tenu de ce qui précède, il faudrait supposer que l'épaisseur du nuage supérieur varie, devenant en certaines zônes, plutôt de l'ordre de 10 que de 100. Par l'analyse de réseaux expérimentaux, on devrait pouvoir déterminer ces variations; et des mesures à plusieurs longueurs d'onde permettraient peut-être de préciser si c'est le sol même, ou un autre nuage plus absorbant, qu'on détecterait ainsi.

\section{Bibliographie}

Bigourd, C., Devaux, C., et Herman, M.: 1973, rapport interne.

Hansen, J. E. et Arking, A.: 1971, Science 171, 669.

Irvine, W. M.: 1968, J. Atmospheric Sci. 25, 610.

Marov, M. Ya., Avduevsky, V. S., Borodin, N. F., Kerzhanovich, V. V., Lysov, V. P., Moshkin,

B. Ye., Rozhdestvenskiy, M. K., Ryabov, O. L., et Ekonomov, A. P.: 1973, Proc. NASA Technical Translation F14, 909.

Wang, L.: 1972, Astrophys. J. 174, 671. 